\title{
The Association of Social Factors and Health Insurance Coverage with COVID-19 Vaccinations and Hesitancy, July 2021
}

\author{
Leighton $\mathrm{Ku}, \mathrm{PhD}, \mathrm{MPH}^{\top} \odot$ \\ ${ }^{1}$ Dept of Health Policy and Management, Milken Institute School of Public Health, George Washington University, Washington, DC, USA
}

Background: There are racial differences in COVID-19 vaccination rates, but social factors, such as lack of health insurance or food insecurity, may explain some of the racial disparities.

Objective: To assess social factors, including insurance coverage, that may affect COVID-19 vaccination as of June-July 2021 and vaccine hesitancy among those not yet vaccinated, and how these may affect racial equity in vaccinations.

Design: Cross-sectional analysis of nationally representative survey data.

Participants: Adults 18 to 64 participating in the Census Bureau's Household Pulse Survey for June 23 to July 5, 2021.

Main Measures: Vaccination: receipt of at least one dose of a COVID-19 vaccine. Vaccine hesitancy: among those not yet vaccinated, intent to definitely or probably not get vaccinated.

Key Results: In unadjusted analyses, black adults were less likely to be vaccinated than other respondents, but, after social factors were included, including health insurance status, food sufficiency, income and education, and state-level political preferences, differences between black and white adults were no longer significant and Hispanics were more likely to be vaccinated $(\mathrm{OR}=1.87, p<.001)$. Among those not yet vaccinated, black and Hispanic adults were vaccine hesitant than white adults (ORs $=.37$ and .45 , respectively, both $p<.001$ ) and insurance status and food insufficiency were not significantly associated with vaccine hesitancy. The percent of state voters for former President Trump in 2020 was significantly associated with lower vaccination rates and with increased vaccine hesitancy.

Discussion: The results indicate that much of the gap in COVID vaccination rates for minority adults are due to social barriers, rather than differences in racial attitudes. Unvaccinated minority adults expressed less vaccine hesitancy than white adults. Social barriers like food insecurity and insurance coverage could have deterred prompt COVID-19 vaccinations. Reducing these problems might help increase vaccination rates.

Received: 14 June 2021

Accepted: 8 October 2021

Published online November 29, 2021
J Gen Intern Med37(2):409-14

DOI: $10.1007 / \mathrm{s} 11606-021-07213-6$

(c) Society of General Internal Medicine 2021

\section{INTRODUCTION}

COVID-19 vaccinations have slowed the progress of the pandemic and may have saved more than 100,000 Americans' lives. ${ }^{1}$ Black and Hispanic adults have been less likely to get COVID-19 vaccinations than white adults, even though racial minorities have experienced higher COVID-19 prevalence and harm. ${ }^{2-4}$ Recent analyses also show that vaccination rates are often lower in areas with greater social vulnerability, such as poverty, unemployment, or higher minority population. ${ }^{5-7}$ Personal characteristics, such as income, education, race/ethnicity, and gender, have been associated with vaccine intentions among the unvaccinated. ${ }^{8}$ Political ideology appears to be involved too; Republicans are less vaccinated and more opposed to vaccination than Democrats. ${ }^{9,10}$

By May 2021, all US adults 18 or older became eligible for free COVID-19 vaccinations. ${ }^{11}$ Before then, COVID vaccines were primarily available to those over 65 , to those with chronic conditions that placed them at risk, and to health care and essential workers, depending on state policies. Beginning in May, vaccines became available to virtually all US adults at no cost. Nonetheless, millions of Americans remain unvaccinated, due to initial vaccine supply shortages, logistical barriers in getting vaccinated, concerns about the safety of vaccines, distrust of the government, and other reasons. The unvaccinated pose a public health hazard both to themselves and to the broader public.

The goal of this study is to more comprehensively examine factors associated with COVID vaccination and vaccine hesitancy, using multivariate analyses to understand the extent to which social and political factors modify differences between racial/ethnic groups. If the social factors like food insufficiency or health insurance coverage reduce the apparent effect of racial/ethnic classifications, this suggests delays in vaccination are more related to structural aspects of racism, including these social barriers, in the USA than to racial or ethnic attitudinal differences. ${ }^{12,13}$ 


\section{METHODS AND DATA}

This article examines how social factors shape vaccination coverage and hesitancy, using data from the Census Bureau's Household Pulse Survey (HPS) for June 23 to July 5, 2021, after all US adults had been vaccine-eligible for 8 weeks. Unlike prior research, which mostly relied on cross-tabulations, this study uses multivariate analyses to examine whether race or ethnicity is still significantly related to vaccination status or intent, after controlling for social factors, such as food insecurity, lack of health insurance, or poverty. This is a cross-sectional analysis of HPS survey data, merged with other data about political environments of the states where respondents lived. Similar analyses were done using data from the earlier April 28-May 10 period, just as vaccines become available to all adults 18-64. Findings of those data are broadly to those reported here; noteworthy exceptions are mentioned. More detailed information about the April 28-May 10 analyses can be requested from the author, upon request.

HPS is an experimental series of rapid response surveys of US adults, representative of the nation and of states, conducted in English and Spanish. ${ }^{14}$ The survey has been widely used to analyze health and social status and trends during the pandemic. ${ }^{15-17}$ The Census Bureau developed survey weights that account for the probability of selection into the sample and non-response; it uses post-stratification adjustments to match Census population estimates for age, race/ ethnicity, and gender by state. ${ }^{18}$ The overall response rate for the June 23-July 10 survey was $6.3 \%$. Analyses of HPS examined the potential for non-response bias and found that response rates were higher among disadvantaged populations, such as those with incomes below poverty or uninsured, compared to other groups. ${ }^{19}$

Respondents were excluded from this analysis if they were 65 or older or had missing insurance coverage data, leaving a sample of 38,504. Those 65 or older were excluded because they were at higher risk if infected and had been eligible for COVID vaccinations since January and because almost all seniors are enrolled in Medicare. To adjust for the survey design and non-response, all bivariate tabular and multivariate logistic regression analyses were conducted using the 80 balanced repeated replicate weights provided by the Census Bureau. The criterion for statistical significance was $p<0.05$ (two-tailed). This study was exempt from human subjects review because it only used secondary, de-identified data.

Key questions about COVID-19 vaccination, intent, and diagnosis are as follows: "Have you received a COVID-19 vaccine?" (yes/no). Those who were not vaccinated (at least one dose) were asked: "Once a vaccine to prevent COVID19 is available to you, would you: Definitely get..., Probably get..., Be unsure about getting..., Probably not get... or Definitely not get a vaccine." In this study, those who were probably or definitely not going to get vaccinated are classified as "vaccine hesitant," the criterion used in a recent federal report. ${ }^{21}$ All were asked: "Has a doctor or other health care provider ever told you that you have COVID-19?" (yes/no/ not sure).

The multivariate statistical models adjust for age, gender, race/ethnicity, marital status, number of children, education, pre-pandemic 2019 income (in eight categories like under $\$ 25,000, \$ 25,000$ to $\$ 34,999)$, loss of employment income in the past 4 weeks, essential work status (whether the respondent worked outside the home during 2021 in one of 15 job categories, such as health care, food production or sales, public transit), being uninsured, and food insufficiency (sometimes or never having enough food to eat in the last 7 days, a relatively severe form of food insecurity). Health insurance status is based on whether the adult had public insurance (e.g., Medicaid), had private insurance only, or was uninsured. For brevity, non-Hispanic racial groups are described as "white," "black," "Asian," and "other." HPS did not ask about political ideology, but a proxy measure was developed: the percent of the popular vote in the 2020 Presidential election for the Republican candidate (Trump) in the respondent's state. ${ }^{20}$ This is not a measure of the individual's political preferences; it indicates the political environment where they reside. The social factors included were selected based on prior research. ${ }^{5-10,21}$

\section{RESULTS}

Table 1 presents weighted bivariate data about vaccination status and, among those not yet vaccinated, hesitancy (definitely or probably will not get vaccinated), classified by

Table 1 Receipt of COVID-19 Vaccinations and, Among Those Not Yet Vaccinated, Intent to Definitely or Probably Not Get Vaccinated, Adults 18-64, June 23-July 5, 2021

\begin{tabular}{llllll}
\hline & Already vaccinated & \multicolumn{2}{l}{$\begin{array}{l}\text { Vaccine hesitant, } \\
\text { among those not } \\
\text { yet vaccinated }\end{array}$} \\
\cline { 2 - 3 } \cline { 5 - 6 } & Percent & $p$ & & Percent & $p$ \\
\hline Overall total & $78.5 \%$ & & $56.8 \%$ & \\
White non-Hispanic & $78.8 \%$ & & $65.8 \%$ & $<0.001$ \\
Black non-Hispanic & $68.7 \%$ & $<0.001$ & & $39.5 \%$ & $<0.001$ \\
Asian non-Hispanic & $95.2 \%$ & $<0.001$ & & $29.4 \%$ & 0.001 \\
Other race non-Hispanic & $72.9 \%$ & $<0.001$ & $60.3 \%$ & \\
Hispanic & $79.3 \%$ & & & $42.8 \%$ & $<0.001$ \\
2020 state election status & & & & & \\
$\quad$ Majority Republican & $72.1 \%$ & $<0.001$ & $60.0 \%$ & 0.01 \\
$\quad$ Minority Republican & $82.6 \%$ & & $53.6 \%$ & \\
Sample size & 38,504 & & 5,881 & \\
\hline
\end{tabular}

Note: Significance for race compares those in group vs those not in group (e.g., Hispanic vs non-Hispanic). Significance for 2020 election status compares those living in states where a majority voted for the Republican Presidential candidate vs a minority

Source: Household Pulse Survey, June 23-July 4, 2021 
racial/ethnic group and political environments for adults 18 to 64. Overall, over two-thirds (78.5\%) received at least one dose of a COVID-19 vaccine by the survey date, but half $(56.8 \%)$ of those not yet vaccinated were vaccine hesitant (definitely or probably not get vaccinated); this represents $7.1 \%$ of total adults in the sample. (An additional $27.0 \%$ were unsure about getting vaccinated.)

Black and other race adults were less likely to be vaccinated than other adults and there were no significant differences between white and Hispanic adults. In analyses of the April 23-May 10 period (not shown), $72.1 \%$ of adults were vaccinated; vaccination rates were lower for black, Hispanic, and other race adults; $44.4 \%$ of those not vaccinated were vaccine hesitant. More people gained vaccinations in the May-June period, but a large share of the unvaccinated remained vaccine hesitant.

Table 2 shows that $14.2 \%$ of adults said they had been told by a physician or other health professional that they had COVID-19, while $85.8 \%$ said they were not infected or were not sure. (The not infected and not sure categories were combined; only $0.8 \%$ said they were not sure about their status.) The vaccination rate was higher among those not previously infected $(80.0 \%)$ than among those who knew they had been infected before $(69.8 \%, p<0.001)$. Prior COVID infection was not significantly associated with vaccine hesitancy, however.

Table 3 presents results of multivariate logistic regression models about vaccination status at the time of the survey and vaccine hesitancy among those not yet vaccinated. As seen in the first column, after controlling for multiple factors, the odds of being vaccinated were higher among Hispanic ( $\mathrm{OR}=1.87, p<0.001)$ and Asian $(\mathrm{OR}=4.95$, $p<0.001$ ) adults compared to white adults, but did not differ significantly between black, other race, and white adults. Vaccinations were significantly higher among those who were older, were more educated, or had higher pre-pandemic
Table 2 Weighted Distribution of Vaccination Status and Intent for Adults 18-64 by Prior COVID-19 Diagnosis Status

Table 3 Odds Ratios for Receiving at Least One Dose of COVID-19 Vaccine or, Among Those Not Vaccinated, Definitely or Probably Not Planning to be Vaccinated, Adults 18-64

\begin{tabular}{llll}
\hline & $\begin{array}{l}\text { Had COVID } \\
\text { diagnosed }\end{array}$ & $\begin{array}{l}\text { Did not have COVID } \\
\text { or not sure }\end{array}$ & $p$ \\
\hline Percent who had COVID diagnosed & $14.2 \%$ & $85.8 \%$ & $<0.001$ \\
$\begin{array}{l}\text { Percent vaccinated (at least one dose), by prior } \\
\text { COVID status }\end{array}$ & $69.6 \%$ & $80.0 \%$ & NS \\
\begin{tabular}{l} 
Percent vaccine hesitant, by prior COVID status \\
\hline
\end{tabular}
\end{tabular}

Source: Household Pulse Survey, June 23-July 5, 2021

\begin{tabular}{|c|c|c|c|c|}
\hline & \multicolumn{2}{|c|}{ Already vaccinated } & \multicolumn{2}{|c|}{$\begin{array}{l}\text { Vaccine hesitant, } \\
\text { among those not yet } \\
\text { vaccinated }\end{array}$} \\
\hline & Odds ratio & $p$ & Odds ratio & $p$ \\
\hline Had COVID diagnosed & 0.611 & & 0.815 & \\
\hline Age (years) & 1.025 & $<0.001$ & 1.004 & \\
\hline Female (vs male) & 1.051 & & 0.756 & 0.004 \\
\hline \multicolumn{5}{|l|}{ Race/ethnicity (vs white non-Hispanic) } \\
\hline Black non-Hispanic & 0.828 & & 0.371 & $<0.001$ \\
\hline Asian non-Hispanic & 4.952 & $<0.001$ & 0.286 & 0.001 \\
\hline Other, multi-race non-Hispanic & 1.002 & & 0.831 & \\
\hline Hispanic & 1.866 & $<0.001$ & 0.450 & $<0.001$ \\
\hline Education (years) & 1.322 & $<0.001$ & 1.031 & \\
\hline Divorced (vs not divorced) & 0.762 & $<0.001$ & 0.935 & \\
\hline Number of children & 0.751 & $<0.001$ & 1.183 & $<0.001$ \\
\hline Essential worker outside home & 0.887 & 0.04 & 1.282 & 0.019 \\
\hline Income in 2019 (categories) & 1.115 & $<0.001$ & 1.136 & 0.001 \\
\hline Lost work income in last 4 weeks & 0.909 & & 0.947 & \\
\hline \multicolumn{5}{|l|}{ Insurance coverage (vs uninsured) } \\
\hline Public insurance & 1.311 & 0.047 & 1.146 & \\
\hline Private insurance only & 1.595 & $<0.001$ & 1.059 & \\
\hline Insufficient food (vs sufficient) & 0.683 & $<0.001$ & 0.780 & \\
\hline Percent of state voting Republican in 2020 & 0.971 & $<0.001$ & 1.013 & 0.027 \\
\hline Sample size & 34,069 & & 5,130 & \\
\hline
\end{tabular}

Source: Household Pulse Survey, June 23-July 5, 2021 
incomes. Those with public insurance (e.g., Medicaid) were more likely to be vaccinated than the uninsured $(\mathrm{OR}=1.31$, $p=0.047)$ as were those with private insurance $(\mathrm{OR}=1.60$, $p<0.001)$. The odds of being vaccinated were lower for those with more children, whose households lost job income in the past four weeks, or who sometimes or often did not have enough to eat in the prior week $(\mathrm{OR}=0.683, p<0.001)$.

The percent of state voters who voted Republican was negatively associated with vaccination $(\mathrm{OR}=0.971, p<0.001)$. That is, living in a state in which the vote for Trump was $10 \%$ higher is associated with $25 \%$ lower odds of being vaccinated $\left(0.971\right.$ to the $10^{\text {th }}$ power $\left.=0.749\right)$. Subanalyses stratified the HPS data for those who lived in states with and without Republican majorities. The effects of the other social factors were similar in both red and blue states; odds ratios for black adults were not significantly different from white adults and being Hispanic was associated with higher odds of being vaccinated in states in both types of states.

In the analyses of the June-July data, having a prior COVID infection was not significantly associated with being vaccinated. However, analyses of the April-May data found that prior infection was associated with lower odds of vaccination $(\mathrm{OR}=0.641, p<0.001)$. The apparent change in the effect of prior COVID diagnoses suggests that many of those who were previously infected did not immediately get vaccinated when vaccines first became available to nonelderly adults in May, but had somewhat caught up by July, 2 months later.

The second column in Table 3 examines vaccine hesitancy among the unvaccinated (probably or definitely not getting vaccinated vs definitely or probably will get vaccinated or are unsure). Black, Hispanic, and Asian respondents were much less likely to be vaccine hesitant, compared to white adults $(\mathrm{ORs}=0.371,0.450$, and 0.286 , respectively). Females were less likely to be hesitant than males $(\mathrm{OR}=0.756, p<0.001)$. Most social factors, such as education, food insufficiency, public or private insurance, and having recent income losses, were not significantly associated with vaccine hesitancy. Prior COVID infection status was not significantly associated with vaccine hesitancy. Those with more children and higher incomes had higher hesitancy. The percent of the state that voted for Trump was associated with increased vaccine hesitancy; a $10 \%$ increase in Republican votes was associated with a $14.2 \%$ increase in the odds of being vaccine hesitant.

\section{DISCUSSION}

These analyses represent a point in time in the COVID-19 vaccination process, as of June 23 to July 5, 2021. Compared to the April 28-May 10 period, there were more vaccinations but the percent of the unvaccinated who oppose vaccination crept up, indicating the challenges of persuading the remaining unvaccinated to change their minds.
While there has been substantial concern about lagging vaccination rates in minority communities, these analyses suggest that after controlling for social factors, such as health insurance and food sufficiency, Hispanics were more likely to be vaccinated than white adults and differences between black and white adults were no longer statistically significant. Moreover, even if they are currently unvaccinated, black, Hispanic, and Asian adults express less vaccine hesitancy than white adults. Social vulnerabilities, like being uninsured, having food insecurity, poverty, and limited education, have been more important factors that impeded vaccination efforts in minority communities, not racial differences in attitudes toward vaccination. Nonetheless, many black and Hispanic adults still have vaccine hesitancy and the need for better outreach and education remains pertinent.

The analyses also confirm the influence of political ideology on attitudes and behaviors related to COVID. ${ }^{9,10}$ Adults living in states that supported President Trump in the 2020 election were much less likely to be vaccinated and more vaccine hesitant. These findings parallel other evidence that Republicans tended to wear masks less often to prevent the spread of COVID. ${ }^{21}$ Evidence also indicates that states with Republican governors have higher COVID19 prevalence and death rates. $^{22}$

Although health insurance is not required for free COVID-19 vaccinations, the uninsured were less likely to be vaccinated than those covered by Medicaid or private insurance. Although COVID vaccinations are free to those without insurance, it is plausible that those who are uninsured are less likely to have a primary care provider who can provide counseling about COVID vaccinations. Further, some uninsured people may not realize that vaccinations are free and worry about the cost.

Other social factors, such as not having enough food to eat, having more children, or being divorced, were also associated with being less vaccinated. These suggest the challenges of survival and caring for children, which can be particularly difficult for single mothers. Those with more longstanding vulnerabilities, such as those with less education or income even before the pandemic, were also less likely to be vaccinated. Some of the specific barriers that socially vulnerable populations may have faced include the lack of internet connections to schedule appointments, lack of transportation, difficulties finding time to get vaccinated due to work or other scheduling challenges, or difficulties finding locations for vaccination that were convenient to them.

A limitation of this analysis is that it is based on crosssectional analysis of survey data and only reports associations; it does not establish causality. The data are based on self-reported characteristics from a survey with a low response rate; they could be subject to sampling bias, reporting error, and missing data. Although a state-level 
measure of the political environment was used, individual data about ideological differences were not available.

Health officials have sought to improve the racial equity of COVID-19 vaccination efforts using multiple approaches, including tailored outreach and messaging to address cultural or information concerns, working with community partners, and providing vaccinations in accessible locations, such as at community health centers, as well as grocery stores and pharmacies. ${ }^{23,24}$ Many communities are already engaging in more active outreach, relying on trusted community members, who may include local health professionals and leaders who share racial, cultural, or political values with their communities. The threat of the new Delta variant and the full FDA approval of COVID vaccines may persuade some more people to enhance their health by becoming vaccinated. Increasing use of vaccine requirements/mandates by the government, employers and schools can also bolster the number who become vaccinated but should be done with further education and a reduction in logistical barriers. ${ }^{25}$ But the persistent pattern of disadvantage and social barriers means that it is also important to take steps to reduce barriers that may impede vaccinations, particularly for black and Hispanic adults. The association of food insecurity with lack of vaccination suggests that offering vaccinations in conjunction with charitable food distribution, such as at food banks or pantries, could be effective. Efforts such as bolstering SNAP benefits to reduce food insecurity ${ }^{26}$ or expanding Medicaid ${ }^{27}$ could also address some of these social barriers and ultimately help improve public health.

Corresponding Author: Leighton Ku, PhD, MPH; Dept of Health Policy and Management, Milken Institute School of Public Health, George Washington University,Washington, DC, USA (e-mail: lku@, gwu.edu).

\section{Acknowledgements}

Leighton $K u$ was the sole author of this study and was responsible for all aspects. The author reports no conflicts of interest regarding this article.

\section{Declarations}

Conflict of Interest The author declares that he/she does not have a conflict of interest.

\section{References}

1. Gupta S, Cantor J, Simon K, et al. Vaccinations Against COVID-19 May Have Averted Up To 140,000 Deaths In The United States. Health Aff (Millwood). 2020: 40(9). Online ahead of print. doi: https://doi.org/10.1377/hlthaff.2021.00619

2. Ndugga N, Pham O, Hill L, et al. Latest Data on COVID-19 Vaccinations Race/Ethnicity. Kaiser Family Foundation. June 9, 2021. https://www.kff.org/coronavirus-covid-19/issue-brief/latestdata-on-covid-19-vaccinations-race-ethnicity/. Accessed June 12,2021 .
3. Baack B, Abad N, Yankey D, et al. COVID-19 Vaccination Coverage and Intent Among Adults Aged 18-39 Years - United States, March-May 2021. Morbid Mortal Wkly Rep; 2021; 70(25):928-33.

4. Santibanez T, Singleton J, Black C, et al. Sociodemographic Factors Associated with Receipt of COVID-19 Vaccination and Intent to Definitely Get Vaccinated, Adults aged $\geq 18$ Years - Household Pulse Survey, United States, April 28-May 10, 2021. Centers for Disease Control and Prevention. June 15, 2021. https://www. cdc.gov/vaccines/imz-managers/coverage/adultvaxview/pubsresources/sociodemographic-factors-covid19-vaccination.html.

5. Barry V, Dasgupta S, Weller D, et al. Patterns in COVID-19 Vaccination Coverage, by Social Vulnerability and Urbanicity - United States, December 14, 2020-May 1, 2021. Morbid Mortal Wkly Rep; 70(22): 818-24.

6. Whiteman A, Wang A, McCain K, et al. Demographic and Social Factors Associated with COVID-19 Vaccination Initiation Among Adults Aged $\geq 65$ Years - United States, December 14, 2020-April 10, 2021 . Morbid Mortal Wkly Rep; 2021; 70(19): 725-30.

7. Hughes MM, Wang A, Grossman MK, et al. County-level COVID19 vaccination coverage and social vulnerability-United States, December 14, 2020-March 1, 2021. Morb Mortal Wkly Rep 2021;70:431-6.

8. Nguyen KH, Srivastav A, Razzaghi H, et al. COVID-19 vaccination intent, perceptions, and reasons for not vaccinating among groups prioritized for early vaccination-United States, September and December 2020. Morb Mortal Wkly Rep 2021 Feb 12; 70(6):217-22.

9. Kates J, Tolbert J, Orgera J. The Red/Blue Divide in COVID-19 Vaccination Rates is Growing. Kaiser Family Foundation. 2021. https://www.kff.org/policy-watch/the-red-blue-divide-in-covid19-vaccination-rates-is-growing/.

10. Frankovic K. Why Won't Americans Get Vaccinated? YouGov. 2021. https://today.yougov.com/topics/politics/articles-repor ts/2021/07/15/why-wont-americans-get-vaccinated-poll-data.

11. National Public Radio, Biden Says All Adults Will Be Vaccine Eligible by April 19. April 6, 2021. https://www.npr.org/sections/ coronavirus-live-updates/202 1/04/06/984745020/biden-willdirect-states-to-make-all-adults-vaccine-eligible-by-april- 19.

12. Khazanchi R, Evans CT, Marcelin J. Racism, Not Race, Drives Inequity Across the COVID-19 Continuum. JAMA Network Open. 2020;3(9):e2019933.

13. Corbie-Smith G. Vaccine Hesitancy Is a Scapegoat for Structural Racism. JAMA Health Forum. 2021 Mar 25;2(3):e210434.

14. Census Bureau. Household Pulse Survey. https://www.census. gov/data/experimental-data-products/household-pulse-survey. html. Accessed June 9, 2021.

15. Cai C, Woolhandler S, Himmelstein D, Gaffney A. Trends in Anxiety and Depression Symptoms During the COVID-10 Pandemic: Results from the US Census Bureau's Household Pulse Survey. $J$ Gen Int Med. 2021; 36(6): 1841-3.

16. Nagata JM, Ganson KT, Whittle HJ, et al. Food Insufficiency and Mental Health in the U.S. During the COVID-19 Pandemic. Am J Prev Med. 2021 Apr;60(4):453-461.

17. Beleche T, Ruhter J, Kolbe A, et al. COVID-19 Vaccine Hesitancy: Demographic Factors, Geographic Patterns, and Changes Over Time. Office of the Assistant Secretary for Planning and Evaluation, HHS. May 2021. https://aspe.hhs.gov/system/files/pdf/ 265341/aspe-ib-vaccine-hesitancy.pdf. Accessed June 12, 2021.

18. Census Bureau. Source of the Data and Accuracy of the Estimates for the Household Pulse Survey - Phase 3.1, Week 33, June 23-July 5, 2021. https://www2.census.gov/programs-surve ys/demo/technical-documentation/hhp/Phase3-1_Source_and_ Accuracy_Week_33.pdf.

19. Peterson S, Toribio N, Farber J. Hornick D. Nonresponse Bias Report for the 2020 Household Pulse Survey. Version 1.0. Census Bureau. March 24, 2021. https://www2.census.gov/progr ams-surveys/demo/technical-documentation/hhp/2020_HPS_ NR_Bias_Report-final.pdf.

20. Cook Report. Final 2020 Election Results. https://cookpolitical. com/2020-national-popular-vote-tracker.

21. Palosky C. Poll: Large Majorities Now Say They Wear Masks Regularly and Can Continue Social Distancing for At Least Six Months if Needed, though Republicans Remain Less Likely to Take Such Precautions. Kaiser Family Foundation. https://www. kff.org/coronavirus-covid-19/press-release/poll-large-major 
ities-now-say-they-wear-masks-regularly-and-can-continuesocial-distancing-for-at-least-six-months-if-needed-though-repub licans-remain-less-likely-to-take-such-precautions /.

22. Neelon B, Mutiso F, Mueller N, et al. Associations Between Governor Political Affiliation and COVID-19 Cases, Deaths, and Testing in the U.S. Am J Prev Med. 2021 July; 61(1):115-119

23. Centers for Disease Control and Prevention. A Guide for Community Partners Increasing COVID-19 Vaccine Uptake Among Members of Racial and Ethnic Minority Communities. April 6, 2021. https://www.cdc.gov/vaccines/covid-19/downloads / guide-community-partners.pdf. Accessed June 12, 2021.

24. Sharac J, Shin P, Ku L, et al. Community Health Centers Move to Frontline Providers of COVID-19 Immunization Services Under President Biden's Health Center COVID-19 Vaccine Program. Geiger Gibson / RCHN Community Health Foundation Research Collaborative Data Note. Feb. 18, 2021. http://gwhpmmatters.com/ community-health-centers-move-frontline-providers-covid-19immunization-services-under-president. Accessed June 10, 2021.

25. Evers-Hillstrom K. Pfizer's full approval triggers new vaccine mandates. The Hill. Aug. 23, 2021. https://thehill.com/policy/healt hcare/569051-pfizers-full-approval-triggers-new-vaccine-manda tes.

26. Adams K, Cunningham R. How does increasing SNAP benefits help low-income families? National Public Radio. Aug. 23, 2021. https: / /www.marketplace.org/2021/08/23/how-does-incre asing-snap-benefits-help-low-income-families /

27. Buchmuller T, Cliff B, Levy H. The Benefits of Medicaid Expansion. JAMA Health Forum. 2020;1(7):e200879.

Publisher's Note Springer Nature remains neutral with regard to jurisdictional claims in published maps and institutional affiliations. 